\title{
The Role of Dynamic Contrast Enhancement MR Imaging as a Modality to Differentiate Between Benign and Malignant Bone lesion
}

\author{
Vivid Umi Varidha $^{\mathrm{a}}$, Paulus Rahardjo ${ }^{\mathrm{b}}$, Rosy Setiawati ${ }^{\mathrm{c}^{*}}$ \\ a vividvaridha02@gmail.com \\ ${ }^{a}$ Resident of Radiology Department, Faculty of Medicine, Airlangga University/Dr.Soetomo General Hospital, Surabaya \\ ${ }^{b}$ Musculosceletal Consultant of Radiology Department, Faculty of Medicine, Airlangga University/Dr.Soetomo General Hospital, \\ Surabaya
}

\begin{abstract}
The study was aimed to determine Time Intensity Curve (TIC) type and slope value on Dynamic Contrast Enhancement Magnetic Resonance Imaging (DCE-MRI) examination technique to differentiate between benign and malignant bone lesion. The study was done on December 2018 to July 2019 with retrospective cross sectional analytic study. The samples were bone lesion patients which was examined with Dynamic Contrast Enhancement on 3T MRI. The sample size were 15 subjects, consisted of $8(53.3 \%)$ males and 7 (46.7\%) females. The age range of subjects was 13 to 75 years old (38,20 \pm 20.49 y.o). Patients with malignant bone lesion were $9(60 \%)$ people and benign bone lesion were $6(40 \%)$ people. DCE-MRI could assess tissue vascularization and perfusion based on TIC. In this study, benign bone lesion showed TIC type 2 and 3, while malignant bone lesion mostly showed TIC type 4, with mean slope value of malignant bone lesion was $83,66 \pm 75,34 \%$ and for benign bone lesion was $9,82 \pm 5,94 \%$ with cut-off value for malignancy of $>26,70 \% / \mathrm{m}$. The mean slope value differential for malignant and benign bone lesion was statistically significant $(\mathrm{p}<0.05)$. TIC examination had sensitivity of $75 \%$ and specificity of $100 \%$ in differentiating malignant and benign bone lesion. The difference in pattern type and mean slope value of TIC between benign and malignant bone lesion showed that DCE-MRI has important role in differentiating between benign and malignant bone lesion.
\end{abstract}

Published by IJRP.ORG. Selection and/or peer-review under responsibility of International Journal of Research Publications (IJRP.ORG)

\section{Background}

Bone tumor is an abnormal growth of new cells of bone tissue, which is more progressive and uncoordinated unlike the normal tissue (Davies et al., 2009). Angiogenesis is an important step in tumor's growth and metastasis. Tumor can only grow within 2-3 mm diameter, based on $\mathrm{O} 2$ diffusion limit in the tissue. In this angiogenesis, the microcirculation component such as arteriole, venule and capillary grow abnormally with incomplete membrane, blurring the normal hierarchy and unorganized, with messed up, winding, fragile and highly permeable blood vessel (Costa et al., 2011; Verstraete and Lang, 2000).

Diagnosis of bone lesion is done comprehensively, consists of history taking, physical examination, radiology and histopathology examination. Radiology examination is an important component in bone lesion diagnosis, that can be done with plain x-ray photo, CT Scanning, MRI Scanning and/or Bone scan, correlating with patient's clinical and demographical condition (Oh et al., 2017; Davies et al., 2009). Conventional MRI 
scanning can evaluate bone lesion morphology, but cannot evaluate the necrosis process and availability of viable cells in the lesion, which are the important information to evaluate therapeutic response and prognosis (Costa et al., 2011; Pilania and Jankharia, 2014; Kawakami et al., 2007). Beside that, conventional MRI has low specificity in differentiating between benign and malignant bone lesion, because many lesions shows unspecific character (Gaa et al., 2017; Greenspan, 2011).

Dynamic Contrast Enhancement MR Imaging (DCE-MRI) is a physiologic imaging method, in which, lesion enhancement is monitored early after intravenous contrast injection (Costa et al., 2011). This technique gives physiological information which cannot be determined with conventional anatomical MR, including the information about vascularization, perfusion, capillary permeability and interstitial volume of a tissue. Tissue with high vascularization and high capillary permeability tends to absorb the contrast early (early enhancement) and more intense than less vascularized tissue (Oh et al, 2017). MR Dynamic Perfusion Contrast qualitatively evaluate bone tumor and tumor-like lesions, based on enhancement pattern, which is shown semi-quantitatively with the evaluation of TIC and quantitatively with pharmacokinetic model determination. This technique has some important advantage (Costa et al., 2011; Zampa et al., 2010), such as:

- Tissue Characterization

- Local Staging

- Identification of active area for guiding the tissue biopsy

- Monitoring of pre-operative chemotherapy

- Detection of recurrent or residual lesion, and differentiating lesion with fibrosis.

During the DCE-MRI examination, the lesion shows rapid early enhancement, followed by plateau or mild washout compared with normal tissue. This is caused by the neoangiogenesis occurred mainly in malignant lesion. The early contrast absorption is caused by the increase in blood vessel, followed by plateau that is caused by accumulation of contrast in the neovasculature and its surrounding interstitium, because of the endothelial permeability change (Pilania and Jankharia, 2014; Lavini et al., 2013).

Benign bone lesion is classified as neoplasm or benign lesion of bone; abnormal growth; genetic defect, and; reactive process. Benign bone tumor occur because the regulation disturbance in normal cells, leading to uncontrolled growth. In the case of infection and inflammation, this increase mainly caused by vasodilation, triggered by recruitment of various proinflammatory molecule, resulting in gradual enhancement pattern. DCE-MRI is the appropriate alternative for in vivo non-invasive evaluation of microvascular tissue (Pilania and Jankharia, 2014; Favad et al., 2012; Li et al., 2009).

\section{Methods}

\subsection{Patients}

This was a cross-sectional study, with retrospective design. The sample size was 15 [8 males, 7 females; age range 13-75 years old (mean 38.20 20.49 y.o)], obtained within study period, which was December 2018 until July 2019. The population was all bone lesion that had been examined with DCE-MRI. The inclusion criteria was the bone lesion which had histopathology examination result. The exclusion criteria was the lesion which DCE cannot determine TIC result, and patient that had been given adjuvant therapy.

Regardless from its benefit in bone lesion evaluation, whether TIC can differentiate between malignant or benign bone lesion is still controversial.1,3,4 Therefore, this study was aimed to determine TIC pattern type in DCE-MRI and determine mean value of TIC slope for each bone lesion. We chose 3 region of interest (ROI) in lesion tissue, which was appropriately chosen. The ROI was placed in appropriate tissue, which was only consists of most enhanced solid component of tissue, excluding the necrotic component or the surrounding normal tissue. The ROI was circle or oval in shape, and there was no special reference for minimum area of ROI. We specified $10 \mathrm{~mm} 2$ as the minimum area for ROI, and $55 \mathrm{~mm} 2$ as the maximum area, to ensure similarity. Three ROI was chosen and ROI placement always compared to DWI and ADC to find the right 
position of viable lesion. TIC interpretation was done blindly by two musculoskeletal consultants with more than 5 years of experience.

After the TIC value for each lesion were determined, we calculated mean \%slope value as well for each bone lesion. Then the final diagnosis was established based on histopathology examination of the lesion.

\subsection{MRI protocol}

The examination protocol used 3T MRI Scanner, Siemens ${ }^{\circledR}$ Magnetom Skyra. After the initial plain MRI was done with standard protocol including coronal, sagittal and axial T1W, T2W and STIR order, DCE-MRI was done. Post-contrast image conventional FST1W was also obtained after DCE-MRI study (Davies et al.,, 2009).

The intravenous contrast agent used in the study was Gadoreric acid $0.5 \mathrm{mmol} / \mathrm{ml}$. Contrast agent (CA) was injected through antecubital vein manually. Single dose of contrast agent $(0.2 \mathrm{mmol} / \mathrm{kg})$ from standard gadolinium was given at the rate of 3-3.5 ml/sec. Injection rate was kept constant for all patients. This procedure was followed by injection of $20 \mathrm{ml}$ normal saline with similar rate with the contrast agent. This procedure was important to flush the drying blood vessel, and to ensure that contrast agent enters the systemic circulation as coherent bolus fluid. DCE-MRI was done with T1 TSE 3D sequence, with 30-40 slices per slab, TR/TE 5.48/1.97 ms, FoV read 200, Fov Phase 100.

\subsection{TIC analysis}

TIC represents tissue response to CA as enhancement value. Physiologic properties that are related to microvascular flow include blood vessel permeability and tissue volume fraction and perfusion. These properties could be determined by curve analysis (Gribbestad, I S et al, 2005) Semiquantitatively physiological perfusion of tumor tissue can be evaluated by calculating the slope of the TIC. Slope is a percent increase in signal intensity per time above the baseline value. Slope shows the initial CA absorption obtained from TIC. Where in the malignant process will occur early enhancement with a high slope compared to the benign process in bone lesions. (J, Cao; L, Xiao et al, 2017)

TIC shows variable vascularization that might overlap benign and malignant lesions based on their enhancement pattern. Malignant lesion commonly shows early rapid enhancement pattern and higher slope compared to benign lesion, although it is not specific (Fayad, Jacob, Wang X, Carrino , \& Bluemke, 2012)

The bone lesion was grouped into 2 groups, benign and malignant bone lesion, and also grouped based on histopathological type.

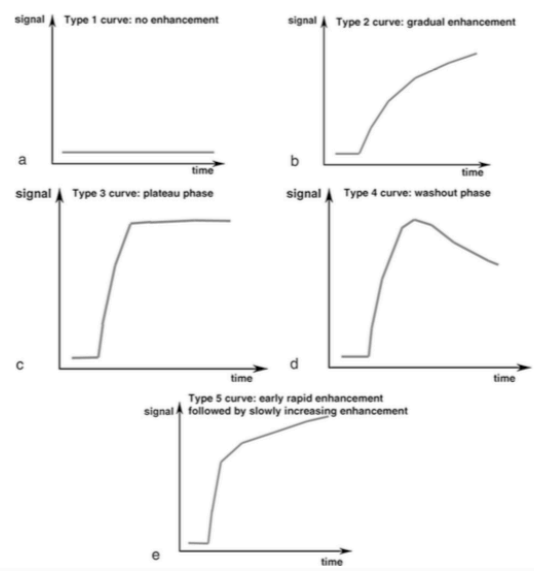

Figure 1. Types of TIC pattern in DCE-MRI examination (Costa et al., 2011). 
The interpretation of the lesion was evaluated based on TIC type and mean \%slope value. TIC was obtained from DCE-FST1W image which can be classified to 5 types (Costa et al., 2011) (Fig.1):

1. Type 1, without enhancement (e.g in lipoma or hematoma)

2. Type 2, gradual enhancement (e.g. in benign lesion)

3. Type 3, rapid early enhancement followed by plateau (limited specificity for benign vascular lesion characterization, desmoid lesion, abscess and several malignant lesion)

4. Type 4, rapid early enhancement followed by washout phase (high vascular lesion with narrow interstitial space, including several malignant lesion, synovial cell sarcoma, leiomyosarcoma, and several benign lesion such as GCT and nidus osteoma)

5. Type 5, rapid early enhancement followed by slow gradual enhancement (e.g in post radiotherapy or chemotherapy lesion, and lesion with wide interstitial space

The Slope value can be calculate by the formula: (Davies, Sundaram , \& James , 2009).

- SImax

$\%$ Slope $=($ SImax - SIbase $) 100 /$ SIbase $($ Tmax - Tbase $)$

- SIbase : Signal intensity before injection of CA Gd-DTPA

- Tmax : First time point at which two \%slope value became $<10 \%$ per minute

- Tbase : time point right before signal intensity enhancement

Mean \%slope value from 3 ROI in the lesion was calculated for vascularization heterogeneity in the lesion (El Backry et al., 2015).

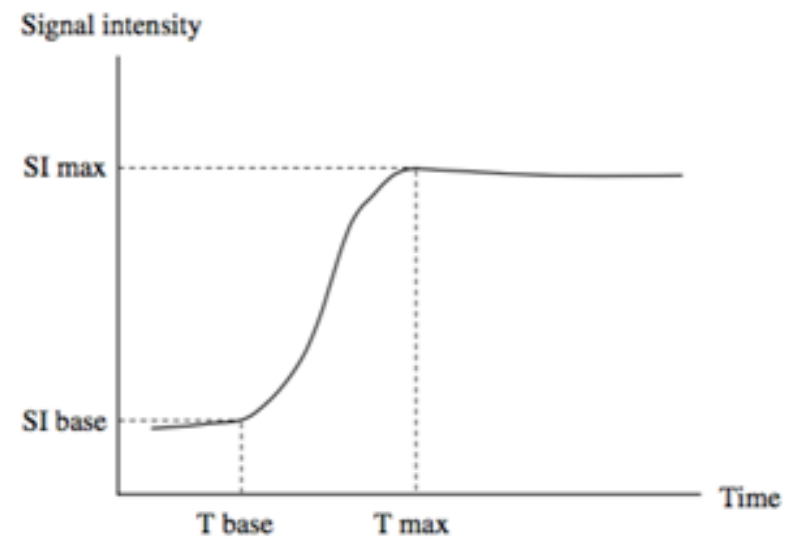

Figure 2. The diagram of Time Intensity Curve, showing SI base, SI maximum, T base and T maximum

2.4. Statistic analysis

For statistical analysis purpose, data analysis was done with statistical software SPSS ${ }^{2}$ 23. Data from DCE-MRI examination of bone lesion produced TIC, followed by calculation of average slope values in each benign and malignant bone lesion groups. All collected TIC type, average slope value, and histopathological results were arranged in tabular form and analyzed. The Mann Whitney test showed that mean \%slope value differential in benign and malignant lesion was statistically significant $(\mathrm{p}<0.05)$.

\section{Results}

The sample size were 15 subjects, consisted of $8(53.3 \%)$ males and $7(46.7 \%)$ females. The age range of subjects was 13 to 75 years old $(38,20 \pm 20.49$ y.o). The final diagnosis showed that $9 / 15(60 \%)$ of the bone 
lesions were malignant and 6/15 (40\%) were benign. The histopathological types of the 36 patients are summarised in Table 1.

Table.1. Patients study

\begin{tabular}{cccccccc}
\hline No. & Name & Sex & Age & Diagnosis & Klasifikasi & $\begin{array}{c}\text { TIC } \\
\text { type }\end{array}$ & $\begin{array}{c}\text { Mean \%slope } \\
\text { value }\end{array}$ \\
\hline 1 & Mrs. K & F & 41 & $\begin{array}{c}\text { Chondroblastic type } \\
\text { Osteosarcoma }\end{array}$ & Malignant & 4 & 54.23 \\
2 & Child P & F & 15 & Osteosarcoma & Malignant & 3 & 118.67 \\
3 & Child J & M & 18 & Osteosarcoma & Malignant & 4 & 32.27 \\
4 & Child I & M & 13 & Osteosarcoma & Malignant & 5 & 69.17 \\
5 & Mr. B & M & 51 & Non Hodgkin & Malignant & 4 & 20.52 \\
6 & Mrs. S & F & 52 & Chondrosarcoma & Malignant & 3 & 49.83 \\
7 & Mr. M & M & 59 & Metastatic Carcinoma & Malignant & 4 & 267.20 \\
8 & Mrs. S & F & 61 & Metastatic Carcinoma & Malignant & 4 & 44.97 \\
9 & Mr. T & M & 58 & Plasmacytoma & Malignant & 3 & 96.10 \\
10 & Mr. M & M & 42 & GCT & Benign & 3 & 14.67 \\
11 & Mr. MZ & M & 30 & GCT & Benign & 3 & 16.27 \\
12 & Mrs. A & F & 26 & GCT & Benign & 3 & 14.23 \\
13 & Mrs. R & F & 19 & GCT & Benign & 2 & 2.92 \\
14 & Child R & M & 13 & Adamantinoma & Benign & 2 & 6.97 \\
15 & Mr. MN & F & 75 & Osteomyelitis & Benign & 2 & 3.83 \\
\hline
\end{tabular}

Table 2. Percentage of lesion type, based on pathological anatomy examination

\begin{tabular}{cccc}
\hline Malignant Lesion & Total (percentage) & Benign lesion & Total (percentage) \\
\hline Osteosarcoma & $4(44.4 \%)$ & Giant Cell Tumor & $4(66.7 \%)$ \\
Non-Hodgkin Lymphoma & $1(11.1 \%)$ & Adamantinoma & $1(16.7 \%)$ \\
Chordosarcoma & $1(11.1 \%)$ & Osteomyelitis & $1(16.7 \%)$ \\
Metastatic carcinoma & $2(22.2 \%)$ & & \\
Plasmacytoma & $1(11.1 \%)$ & Total & 6 \\
Total & 9 & & \\
\hline
\end{tabular}

The TIC type 3 was found in 3 out of 9 malignant bone lesion and 3 out of 6 benign lesion. The TIC type 4 was found in 5 malignant bone lesion, consisted of 2 osteosarcoma, non-Hodgkin lymphoma and 2 metastatic carcinoma. Meanwhile TIC type 5 was found in 1 malignant bone lesion which was osteosarcoma. We showed in table 3 .

Table 3. Pattern type of TIC in DCE-MRI based on bone lesion type

\begin{tabular}{cccc}
\hline TIC Type & Malignant & Benign \\
\hline Type 2 & - & 3 \\
Type 3 & 3 & 3 \\
Type 4 & 5 & - \\
Type 5 & 1 & - \\
\hline
\end{tabular}


The TIC curve and mean \%slope value from 3 ROIs in the lesion was predicted as the evaluation of vascularization, tissue perfusion, endothelial permeability and interstitial space in each ROI (Costa et al., 2011). Table 4 shows mean \%slope value for each lesion in DCE-MRI. Mean \%slope value for malignant bone lesion was $83.66 \pm 75.34 \%$, and for the benign lesion was $9.82 \pm 3.94 \%$ with cut-off value for malignancy of $>26,70 \% / \mathrm{m}$.

Table 4. Mean \%slope value based on pathological anatomy examination results

\begin{tabular}{cccc}
\hline Malignant & Mean $\%$ slope \pm SD & Benign & Mean \%slope \pm SD \\
\hline Osteosarcoma & $68.59 \pm 36.67$ & Giant Cell Tumor & $12.02 \pm 6.13$ \\
Non-Hodgkin Lymphoma & $20.52^{*}$ & Adamantinoma & $6.97^{*}$ \\
Chordosarcoma & $49.83^{*}$ & Osteomyelitis & $3.83^{*}$ \\
Metastatic carcinoma & $156.08 \pm 157.14$ & & \\
Plasmacytoma & $96.10^{*}$ & & $9.82 \pm 5.93$ \\
Total & $83.66 \pm 75.34$ & Total & \\
\hline
\end{tabular}

*Pathological anatomy results with only 1 study subject, no standard deviation

This study also wanted to find out the sensitivity and specificity of TIC as the modality in diagnosing benign and malignant bone lesion. The TIC type 3, 4 and 5 was classified as malignant lesion, and TIC type 1 and 2 was classified as benign lesion. The result was TIC type examination has $75 \%$ sensitivity and $100 \%$ specificity.
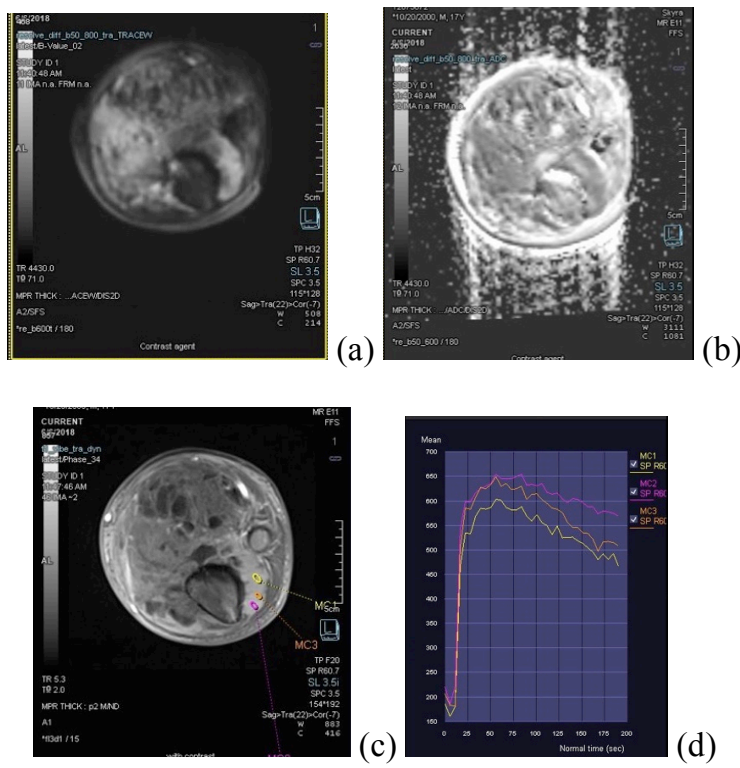

Figure 2. Male, 18 years old, (a) (DWI), (b) (ADC map), (c) (DCE), (d) shows TIC from 3 ROI that was placed at DCE. ROI was placed in most enhanced component of the lesion while comparing to DWI and ADC, where DWI shows restricted diffusion area and reduced ADC. TIC showed type 4 (rapid early enhancement followed by washout phase) and mean slope 32,27\%/m (cut-off value for malignancy of $>26,70 \% / \mathrm{m}$ ), confirmed with pathology examination of osteosarcoma. 

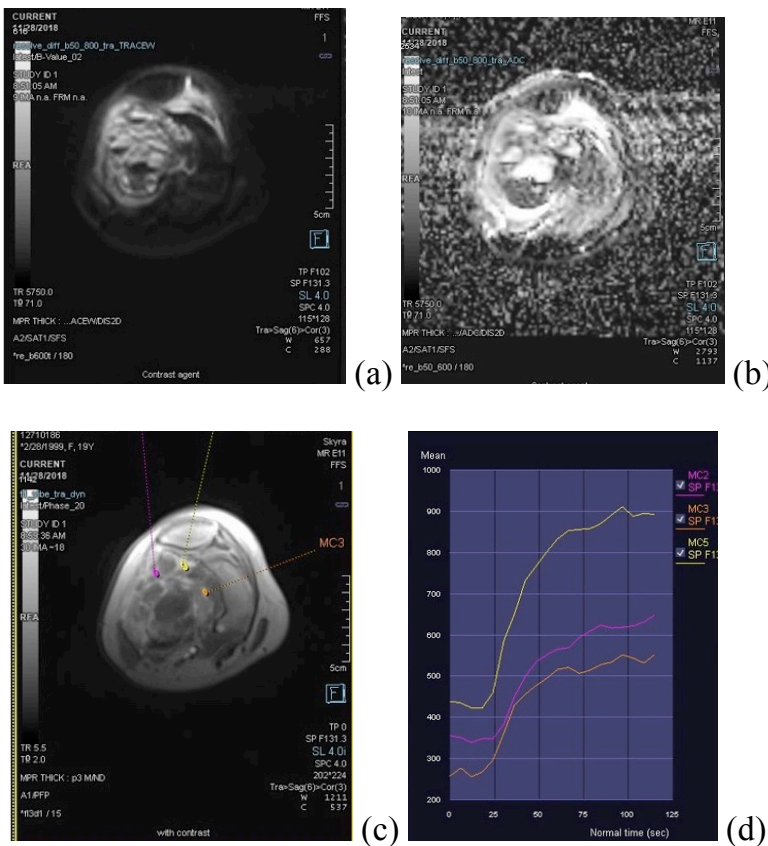

(b)

Figure 3. Female, 19 years old, (a) (DWI), (b) (ADC map), (c) (DCE), (d) shows TIC type 2 (gradual enhancement), and mean slope $2,92 \% / \mathrm{m}(<$ cut-off $6,70 \% / \mathrm{m})$. Confirmed as benign lesion with pathology examination of Giant Cell Tumor

\section{Discussion}

DCE-MRI is a physiological imaging method with low spatial resolution but very high temporal resolution, enabling the study of contrast increase dynamic, during and right after (first-pass) the absorption of gadolinium-chelate to the tissue. According to the Nyquist limit theory, physiologic process must be sampled at twice the frequency of the measured dynamic event. In the first pass phase, usually less than 6-10 seconds, high microvascularization and tissue perfusion of the lesion resulted in early enhancement. In the later phase, capillary permeability and interstitial space enhancement can show plateau phase, washout or post-arterial increase enhancement (Li et al., 2009).

The TIC showed vascularization variation that can overlap between benign and malignant lesion based on its enhancement pattern (van Rijswijk et al, 2004). Generally, malignant lesion shows early rapid enhancement pattern and higher slope compared to benign lesion despite not always specific (Fayad et al., 2012). Previous study showed that TIC type examination has $77 \%$ sensitivity and $78 \%$ specificity in differentiating between benign and malignant lesion, with mean \%slope value for malignant lesion was $70.4 \pm 60.3 \%$, significantly higher than benign lesion, which was $37.6 \pm 52.9 \%$ (Kawakami et al., 2007). Another study by Cao et al. (2017) showed that TIC examination had $95.5 \%$ sensitivity and $85.7 \%$ specificity in differentiating between benign and malignant lesion. Malignant lesion with high vascularization and perfusion showed early enhancement pattern, and after that, there were various pattern possible, depended on capillary permeability and interstitial space. In the lesion with very narrow interstitial lesion showed washout phase (5 seconds), which was TIC type 4 . Meanwhile in the lesion with relatively narrow interstitial space 
showed plateau (2-3 minutes), which was TIC type 3. Finally in the lesion with low cellularity and wide interstitial space showed gradual increase, which was TIC type 5 . The TIC type 5 can be found in malignant lesion which has been given adjuvant chemotherapy or radiotherapy (Li et al., 2009).

In this study, benign bone lesion showed TIC type 2 and 3, and in malignant lesion, mostly showed TIC type 4. The TIC type 3 was found in the lesions with histopathology examination of osteosarcoma, chondrosarcoma, plasmacytoma and GCT, meanwhile TIC type 2 was found in adamantinoma and osteomyelitis, and TIC type 4 was found in osteosarcoma, NHL and metastatic carcinoma. In this study, TIC examination had $75 \%$ sensitivity and $100 \%$ specificity. There was overlapping TIC type in this study, with 3 benign lesion (GCT) showed early enhancement pattern (type 3 ). This finding corresponded with the previous study by van Rijswijk et al. (2004), which they found early enhancement pattern in 32 out of 67 benign lesion. This finding could be affected by the high vascularization of benign lesion, especially GCT. After that, the analysis continued with calculation of mean \%slope value. The result of mean \%slope value for malignant bone lesion was $83.66 \pm 75.34 \%$, and for benign lesion was $(9.82 \pm 5.94 \%)$. The mean \%slope value differential was statistically significant $(\mathrm{p}<0.05)$ with cut-off value for malignancy of $>26,70 \% / \mathrm{m}$.

Giant Cell Tumor, the most common benign lesion in our study, had high vascularization causing the TIC pattern showed similar pattern with malignant lesion, which was early enhancement followed by early washout or plateau, but the mean \%slope value still lower than malignant lesion $(12.02 \pm 6.13 \%)$. This value was the highest from all benign lesion in this study, Study by Kawakami et al. (2007) showed similar results that the GCT showed highest mean \%slope value among all musculoskeletal lesion.

Slope value distribution in malignant bone lesion was significantly higher than benign lesion. This indicated that malignant bone lesion consisted of high vascularization and perfusion area. Therefore, slope value can be used to differentiate between malignant and benign bone lesion. TIC pattern might reflect physiological aspect of tumor tissue, for example, angiogenesis pattern in bone tumors, which might be useful for antiangiogenic agent treatment evauation in the future. Therefore, in the near future this protocol can be implemented in treatment response monitoring include neoadjuvant chemotheray, radiation as well as after surgery.

There was some limitation in this study. First, the participating sample in this study was still few, with little variation of benign bone lesion. Second, ROI placement had some subjective factor. Limiting the placement of ROI to a few area of lesion caused bias in choosing the image.

\section{Conclusion}

Signal intensity change which was monitored by DCE-MRI can be used to evaluate the physiology of bone lesion in terms of vascularization, perfusion, capillary permeability and interstitial space. The difference between TIC pattern type and mean slope value in our study showed that DCE-MRI has important role in differentiating between malignant and benign bone lesion.

We hope that our method, which combined TIC type analysis and slope value, can increase the confidence of radiologists in diagnosing malignant and benign bone lesion.

\section{Acknowledgment}

I would like to dedicate my gratitude to all the lecturers of Radiology, Faculty of Medicine Airlangga university, my husband, my children, my family, and my friends for their endless support and prayers.

\section{References}

Cao, J., Xiao, L., He, B., Zhang, G., Dong, J., Wu, Y., ... \& Lin, X. (2017). Diagnostic value of combined diffusion-weighted imaging with dynamic contrast enhancement MRI in differentiating malignant from benign bone lesions. Clinical radiology, 72(9), 793-e1.

Chockalingam, A., Duran, R., Sohn, J. H., Schernthaner, R., Chapiro, J., Lee, H., ... \& Lin, M. (2016). Radiologic-pathologic analysis of 
quantitative 3D tumour enhancement on contrast-enhanced MR imaging: a study of ROI placement. European radiology, 26(1), 103113.

Choyke, P. L., Dwyer, A. J., \& Knopp, M. V. (2003). Functional lesi imaging with dynamic contrast-enhanced magnetic resonance imaging. Journal of Magnetic Resonance Imaging, 17(5), 509-520.

Costa, F. M., Canella, C., \& Gasparetto, E. (2011). Advanced magnetic resonance imaging techniques in the evaluation of musculoskeletal tumors. Radiologic Clinics, 49(6), 1325-1358.

Davies, A. M., Sundaram, M., \& James, S. J. (Eds.). (2009). Imaging of bone tumors and tumor-like lesions: techniques and applications. Springer Science \& Business Media.

Drapé, J. L. (2013). Advances in magnetic resonance imaging of musculoskeletal tumours. Orthopaedics \& Traumatology: Surgery \& Research, 99(1), S115-S123.

El Backry, M., Shady, M., Mousa, A. E., \& Zaky, M. M. (2015). Role of dynamic contrast enhanced MR perfusion in differentiation between benign and malignant tumors. The Egyptian Journal of Radiology and Nuclear Medicine, 46(3), 715-726.

Fayad, L. M., Jacobs, M. A., Wang, X., Carrino, J. A., \& Bluemke, D. A. (2012). Musculoskeletal tumors: how to use anatomic, functional, and metabolic MR techniques. Radiology, 265(2), 340-356.

Greenspan, A. (2011). Radiologic Evaluation of Tumors and Tumor- like Lesions. In Orthopaedic Imaging a Practical Approach. Lippincott Williams \& Wilkins. Philadelphia.

Gribbestad, I S et al. (2005). An Introduction To Dynamic Contrast- Echanced MRI In Oncology. Springer.

J, Cao; L, Xiao et al. (2017). Diagnostic Value Od Combined Diffusion-weighted Imaging With Dynamic Contrast Enhancement MRI In Differentiating Malignant From Benign Bone Lesions. Clinical Radiology 72, 793.el - 793.e9.

Kawakami, Y., Kunisada, T., Sugihara, S., Ono, A., Nishida, K., Abe, N., ... \& Ozaki, T. (2007). New approach for assessing vascular distribution within bone tumors using dynamic contrast-enhanced MRI. Journal of cancer research and clinical oncology, 133(10), 697.

Lavini, C., Buiter, M. S., \& Maas, M. (2013). Use of dynamic contrast enhanced time intensity curve shape analysis in MRI: theory and practice. Reports in Medical Imaging, 6, 71-82.

Li, X., Springer Jr, C. S., \& Jerosch- Herold, M. (2009). First- pass dynamic contrast- enhanced MRI with extravasating contrast reagent: evidence for human myocardial capillary recruitment in adenosine- induced hyperemia. NMR in Biomedicine: An International Journal Devoted to the Development and Application of Magnetic Resonance In vivo, 22(2), 148-157.

Nussenbaum, F., \& Herman, I. M. (2010). Tumor angiogenesis: insights and innovations. Journal of oncology, 2010.

O'Connor, J. P. B., Tofts, P. S., Miles, K. A., Parkes, L. M., Thompson, G., \& Jackson, A. (2011). Dynamic contrast-enhanced imaging techniques: CT and MRI. The British journal of radiology, 84(special issue 2), S112-S120.

Oh, E., Yoon, Y. C., Kim, J. H., \& Kim, K. (2017). Multiparametric approach with diffusion-weighted imaging and dynamic contrastenhanced MRI: a comparison study for differentiating between benign and malignant bone lesions in adults. Clinical radiology, $72(7), 552-559$.

Pilania, K., \& Jankharia, B. (2014, March). Mean curve analysis of pattern of enhancement of various bone tumors on high resolution dynamic contrast enhanced MR and its role in characterizing various bone tumors. European Congress of Radiology 2014.

Reddick, W. E., Taylor, J. S., \& Fletcher, B. D. (1999). Dynamic MR imaging (DEMRI) of microcirculation in bone sarcoma. Journal of Magnetic Resonance Imaging: An Official Journal of the International Society for Magnetic Resonance in Medicine, 10(3), 277-285.

van Rijswijk, C. S., Geirnaerdt, M. J., Hogendoorn, P. C., Taminiau, A. H., van Coevorden, F., Zwinderman, A. H., ... \& Bloem, J. L. (2004). Soft-tissue tumors: value of static and dynamic gadopentetate dimeglumine-enhanced MR imaging in prediction of malignancy. Radiology, 233(2), 493-502.

Verstraete, K. L., \& Lang, P. (2000). Bone and soft tissue tumors: the role of contrast agents for MR imaging. European journal of radiology, 34(3), 229-246.

Zampa, V., Roselli, G., \& Beltrami, G. (2010). MRI of bone tumors: advances in diagnosis and treatment assessment. Imaging in Medicine, 2(3), 325-340. 